\title{
TECHNIK UND KULTUR ALS MEDIEN DER NACHHALTIGKEIT - GIBT ES EINEN NEXUS VON HUMANÖKOLOGIE, PHILOSOPHISCHER ANTHROPOLOGIE UND GESELLSCHAFTSTHEORIE?
}

Die Fülle an Thematisierungsstrategien und Reflexionsrahmungen, die sich auf die Erschließung des Verhältnisses von Kultur und Technik richten, spiegelt die Komplexität der wechselseitigen Bedingtheiten und Beeinflussungen wider, mit der beide Phänomene aufeinander einwirken. So weit sich das Feld der Erörterungen von Kultur und Technik also auch erstrecken mag, es ist davon auszugehen, dass der Zusammenhang von Theorie und Praxis, der ihnen zu Grunde liegt, einen sinnvollen Diskurs zwischen allen Ansätzen ermöglicht, unabhängig davon, von wo aus, wie und mit welchen Mitteln sie dieses Verhältnis gedanklich erschließen. Sicher ist daher, dass es sowohl erforderlich als auch möglich ist, seine Aufmerksamkeit auf bestimmte Ausschnitte oder charakteristische Verbindungen zu konzentrieren, ohne - auf der substantiellen Ebene - Gefahr zu laufen, keinen wesentlichen Zugang zu diesem Verhältnis zu finden, und ohne - auf der diskursiven Ebene - die nötige Anschlussfähigkeit zu verlieren. Im vorliegenden Beitrag wird die Erörterung des Verhältnisses zwischen Kultur und Technik als Medien der Nachhaltigkeit in einer dreistufig angelegten Vorgehensweise entfaltet. Erstens wird eine Thematisierungsstrategie und Reflexionsrahmung nachvollzogen, die mit den Mitteln der begrifflichen Explikation von „Kultur“ und „Technik“ arbeitet und die wechselseitigen Bezugnahmen der Bedeutungsmomente und Sinngehalte dieser Begriffe ermittelt. Zweitens geht es um eine andere Thematisierungsstrategie und Reflexionsrahmung, die danach fragt, wie Kultur und Technik entstanden sind, und welche Funktionen sie erfüllen, um vor diesem Hintergrund ihr Verhältnis zu entschlüsseln. Drittens werden Kultur und Technik mit Blick auf ihnen zugerechnete Eigenschaften als Zwecke zu gelten bzw. als Mittel zu fungieren hinterfragt und als „Medien“ menschlicher Selbstverwirklichung charakterisiert.

Stichworte: Mensch, Leben, Welt, Natur, Gesellschaft, Interaktion, Kommunikation, Sinn, Metabolismus

\section{EINLEITUNG}

Die Fülle an Thematisierungsstrategien und Reflexionsrahmungen, die sich auf die Erschließung des Verhältnisses von Kultur und Technik richten, spiegelt die Komplexität der wechselseitigen Bedingtheiten und Beeinflussungen wider, mit der beide Phänomene aufeinander einwirken. So weit sich das Feld der Erörterungen von Kultur und Technik also auch erstrecken mag, es ist davon auszugehen, dass der Zusammenhang von Theorie

\footnotetext{
${ }^{1}$ Prof. dr. Andreas Metzner-Szigeth, Professur für Nachhaltigkeitswissenschaft, Institut für Bildung, Kultur und Nachhaltige Entwicklung (IBKN), Hochschule Bochum - University of Applied Sciences, Lennershofstrasse 140, D - 44801 Bochum, e-mail: andreas.metzner-szigeth@hsbochum.de
} 
und Praxis, der ihnen zu Grunde liegt, einen sinnvollen Diskurs zwischen allen Ansätzen ermöglicht, unabhängig davon, von wo aus, wie und mit welchen Mitteln sie dieses Verhältnis gedanklich erschließen.

Sicher ist daher, dass es sowohl erforderlich als auch möglich ist, seine Aufmerksamkeit auf bestimmte Ausschnitte oder charakteristische Verbindungen zu konzentrieren, ohne - auf der substantiellen Ebene - Gefahr zu laufen, keinen wesentlichen Zugang zu diesem Verhältnis zu finden, und ohne - auf der diskursiven Ebene - die nötige Anschlussfähigkeit zu verlieren. Daher ist es erfolgversprechend mein Thema im Verlauf einer dreistufig angelegten, mitunter etwas sprunghaft verfahrenden Vorgehensweise zu entfalten.

\section{KULTUR \& TECHNIK: BEGRIFFLICHE DIMENSIONEN UND GESELLSCHAFTLICHE PRAXIS}

\subsection{Gedanken zur begrifflichen Bestimmung von Kultur}

Um der Weite dieses Phänomens gerecht zu werden, ist es unumgänglich, zumindest zwischen einem „ethnologischen“ und einem „humanistischen“ Begriff des Kulturellen zu unterscheiden. Während der erste deskriptiv auf alle Aspekte einer Lebensweise zielt, auf das Selbst- und Weltverständnis eines Volkes inklusive seiner Bräuche und Werte, ist der zweite normativ $\mathrm{zu}$ verstehen, stellt Kultur etwas durch Bildungsprozesse Hervorgebrachtes und im Sinne einer fortschreitenden Zivilisierung Hervorzubringendes dar.

Zwei essenzielle Komponenten jeden Kulturbegriffs sind erstens die Annahme des Vorhandenseins von sowohl räumlich wie zeitlich begrenzten, ideellen wie materiellen „Mustern“ und zweitens die Annahme eines Nexus zwischen der Idee von Kultur als einem Subjekt, das etwas bewirkt, und der Idee von Kultur als einem Objekt, auf das etwas einwirkt. Zusammen ergibt dies die Eigenschaft, sowohl Produkt von Handlungen als auch konditionierendes Element weiterer Handlungen (jeweils inklusive kommunikativer Akte) zu sein.

Gerade in diesem Sinne ist Kultur mehr als die Menge der Elemente eines unscharf ausdifferenzierten gesellschaftlichen Bereichs, der darauf spezialisiert wurde, kulturelle „Güter“ zu produzieren und in dem „Kulturschaffende“ tätig sind, in Kunst, Musik und Literatur. Sie stellt in diesem besonderen Sinne aber auch mehr dar, als einfach Lebensstile oder auch eine Menge von Normen, Werten oder Überzeugungen. Vielmehr ist sie als eine Art von Matrix zu verstehen, die Bedeutungsfelder ausweist, die bestimmte Assoziationsmöglichkeiten bietet (und andere ausschließt), die sinnstiftende Begründungen für distinkte Handlungsweisen und Interaktionsmuster bereithält, die miteinander verbunden deskriptiv und präskriptiv arbeitet, also kognitiv richtiges (,funktionales“) und normativ richtiges (,gutes“) Handeln (oder besser: Opportunitäten des Handelns) ausweist und in diesem Sinne Wirklichkeit (um-)gestaltende Praxis ist.

\subsection{Gedanken zur begrifflichen Bestimmung von Technik}

Um der Breite dieses Phänomens gerecht zu werden, ist es unabdingbar, zumindest zwischen den Bedeutungen der artefaktischen Technik, des technischen Handelns, des technischen Wissens und der Technikkultur zu unterscheiden.

Dies spricht zunächst für ein Verständnis, in dem Technik erstens die Menge der nutzenorientierten, künstlichen, gegenständlichen Gebilde umfasst, zweitens die Menge 
menschlicher Handlungen, in denen Sachsysteme entstehen, und drittens die Menge menschlicher Handlungen, in denen Sachsysteme verwendet werden ${ }^{2}$. „Technik“ umfasst so nicht nur die von Menschen gemachten Gegenstände („Artefakte“) selbst, sondern schließt auch deren Entstehungs- und Verwendungszusammenhänge („Kontexte“) ein (also ihr „Gemacht-Sein“ und ihr „Verwendet-Werden“).

Darüber hinaus ist anzumerken, dass hier keine systematische Unterscheidung von „Technik“ und „Technologie“ nachvollzogen wird, erstens, weil sie im außerdeutschen Sprachraum keine Rolle spielt, und zweitens, weil sowieso komplexere Unterscheidungen erforderlich sind, nämlich mindestens die zwischen den Bedeutungen der artefaktischen Technik, des technischen Handelns, des technischen Wissens und der Technikkultur. Dies hat u. a. den Vorteil, dass die Komponente des technischen Wissens jedwede Thematisierung von Technologie im Sinne einer Wissenschaft der Technik ${ }^{3}$ ermöglicht, aber auch alle anderen Formen von Technikwissen inkludiert.

Insofern die „Nützlichkeit von Technik [...] immer auch etwas kulturell Interpretiertes " ${ }^{4}$ ist, ist weiter einzusehen, dass Kultur den Entwurf und die Implementierung technischer Lösungen (für sozio-kulturell ausbuchstabierte Zwecke) ebenso erheblich beeinflusst wie deren Aneignung und Nutzbarmachung durch ihre Verwender. Auf beiden Seiten der „Schnittstelle“ von Technik und Kultur bilden sich daher Komplemente, einerseits „Technikkulturen“, andererseits „Kulturtechniken“.

Grundsätzlich ist hier zu berücksichtigen, dass sich parallel zur Entwicklung neuer Fragestellungen und Arbeitsfelder ein Perspektivenwechsel im Verständnis der Technik vollzogen hat, dem gegenüber es darauf ankommt, die essentiell wichtigen Teile beider Perspektiven angemessen aufnehmen zu können. Ausgehend von der einen - früher dominanten - Perspektive werden Technik und Gesellschaft als etwas wesentlich Getrenntes wahrgenommen, als Phänomene, die sich in der Welt vergleichsweise fremd gegenüberstehen, und zwar stark, doch nur äußerlich aufeinander einwirken. Dieser Sichtweise entsprechend wird die Technik als durch ihre Gegenständlichkeit und eigene Gesetzmäßigkeit geprägt gesehen. Ausgehend von der anderen - heute (zumindest bereichsweise) dominanten - Perspektive wird der Blick auf den inneren Zusammenhang von Technik und Gesellschaft gerichtet, der im iterativen Prozess der gesellschaftlichen Hervorbringung von Technik gesehen wird, die als Hervorgebrachtes formativ innerhalb des gesellschaftlichen Entwicklungsgeschehens wirkt. Dieser Sichtweise entsprechend wird die Technik als Prozess technischen Handelns begriffen, der in seinem Verlauf und seinen Ergebnissen als ein durch die Logik des Sozialen bestimmter angesehen wird. Verkürzt betrachtet steht damit auf der einen Seite das Naturale und (Arte-)Faktische der Technik im Vordergrund, die als bestimmende Größen ihres Seins angenommen werden, während auf der anderen Seite das Kulturale und sozial Konstruierte der Technik im Vordergrund stehen, so dass angenommen wird, diese seien die bestimmenden Größen des technischen Geschehens. Dabei wird Technik dem ersten Sinne nach als (naturgesetzlich bestimmtes) Mittel für sozial gesetzte Zwecke betrachtet, die in ihre

\footnotetext{
${ }^{2}$ Ropohl, Günter (1993): Technik. In: Brockhaus-Enzyklopädie. Bd. 21. Mannheim, S. 672-674

3 Vgl. Banse, Gerhard (Hg.) (1997): Allgemeine Technologie zwischen Aufklärung und Metatheorie. Johann Beckmann und die Folgen. Berlin.

${ }^{4}$ Hörning, Karl Heinz (1985): Technik und Symbol - Ein Beitrag zur Soziologie alltäglichen Technikumgangs. In: Soziale Welt, H. 2, S. 185-207
} 
dingliche Gestalt und Funktionsweise eingearbeitet werden. Im zweiten Sinne wird die Technik als ein Medium sozialer Handlungs- und Kommunikationsprozesse verstanden, das genuin gesellschaftlichen Charakters ist und daher nur von sozialwissenschaftlich zu erschließenden Gesetzmäßigkeiten bestimmt ist.

\subsection{Vergleiche und Verbindungen}

Weder ist Technik Kultur, noch ist Kultur Technik - das wären falsche „Gleichsetzungen“, die dem je Besonderen beider Phänomene nicht gerecht würden. Durchaus möglich sind aber der „Vergleich“ von Technik mit (bzw. gegenüber) Kultur und von Kultur mit (bzw. gegenüber) Technik, um beides, dass ihnen Gemeinsame und dass sie Trennende klarer herauszuarbeiten.

Noch besser erscheint mir, gerade auch um die Dimensionen der Begriffe herauszuarbeiten, um die es hier geht, sie als Knoten eines semantischen Netzes zu begreifen. Zwecks Vereinfachung kann hierbei anfangs so vorgegangen werden, jeweils zwei von ihnen als Gegensatzpaare zu behandeln, also etwa, um zunächst einen größeren Bogen zu schlagen, Kultur und Natur, Kultur und Gesellschaft, Technik und Natur, sowie Technik und Gesellschaft, um dann, im engeren Zusammenhang, das Verhältnis von Kultur und Technik im Zusammenhang des Verhältnisses von Natur und Gesellschaft zu bearbeiten. Allerdings stellen diese Oppositionen nur ein Verfahren dar, um bestimmte Bereiche des Netzes mit hoher Auflösung ,abtasten“ zu können.

Erleichtert wird diese Herangehensweise durch die ebenso auffällige wie bedeutsame Feststellung, dass wir es hier wesentlich mit Korrelatbegriffen zu tun haben, also solchen, die nur durch Bezug auf einen Gegenbegriff näher bestimmt werden (können). Das Paradebeispiel dafür ist die Kultur, die alles das umfasst, was nicht Natur ist, die ihrerseits all das ist, was ,von Natur aus“" so ist, wie es ist, also nicht Kultur ist ${ }^{5}$.

Obwohl nun (in einem ontologischen Sinne) letztlich nicht zu klären ist, was denn Kultur und Natur substantiell (d. h. für sich genommen) sind, und wie sich die Erkenntnis des einen sauber von der des anderen trennen ließe, sind die Begriffe Natur und Kultur damit nicht sinnlos: sie sind nur aufeinander Bezug nehmend (antinomisch) zu bestimmende, relationale Kategorien (Konstrukte), die den (im Einzelnen) zu untersuchenden Erfahrungen und Problemen vorausgehen ${ }^{6}$.

Vergleicht man Kultur mit (bzw. gegen) Gesellschaft, so gewinnt man einen Kulturbegriff im engeren Sinne: die „höhere“ Kultur, die sich etwa in Kunst, Musik und Literatur äußert, findet hierbei ihren Ausdruck in nicht-zweckorientiertem Handeln. Gesellschaft hingegen wird mit zweckmäßigem, „niederen“ Tun verbunden und meist der „naturnotwendigen“ Sphäre der Sozioökonomie gleichgesetzt.

Vergleicht man hingegen Kultur mit (bzw. gegen) Natur, so gelangt man zu einem Kulturbegriff im weiteren Sinne: Kultur wird mit Gesellschaft gleichgesetzt, sie ist soziale, kommunikative und technische, materielle Kultur.

\footnotetext{
${ }^{5}$ Gedö, Andras (1986): Die Geschichtlichkeit des Naturbegriffs. In: Dialektik, Bd. 12, S. 99-114; Laitko, Hubert (1986): Natur - Zur Entwicklung einer philosophischen und wissenschaftlichen Kategorie. In: Dialektik, Bd. 12, S. 115-130.

${ }^{6}$ Vgl. auch Böhme, Gernot (1992): Natürlich Natur. Über Natur im Zeitalter ihrer technischen Reproduzierbarkeit. Frankfurt am Main; vgl. hierzu auch Hubig, in diesem Band.
} 
Deutlicher wird dies unter Hinzuziehung des Vergleichs von Natur und Gesellschaft: Natur wird hierbei zum unabhängig vom Menschen so Seienden, wird verstanden als vom Menschen unbeeinflusstes Dasein gleich Natur-Sein. Gesellschaft hingegen wird so zum Produkt des Menschen, zum artifiziellen Dasein, zum so (und nicht anders) gestalteten und gemachten.

Es ist aber zu bezweifeln, ob die Implikation, dass nämlich das Gesellschaftliche dem menschlichen Akteur restlos verfügbar ist, da es von ihm hervorgebracht wird, auch tatsächlich stimmen kann. Schließlich ist das Gesellschaftliche von den Akteuren nicht hinterschreitbar - sie selbst sind gesellschaftlich konstituierte Größen. Nicht weniger zu bezweifeln ist, ob ein Naturbegriff stimmen kann, der sie als unabhängig vom Menschen so Seiendes fasst, denn schließlich fällt der Mensch selbst, als Naturwesen, in den Zusammenhang des Natürlichen, der von ihm ebensowenig hinterschreitbar ist.

\subsection{Verständnisse und Verwendungen}

Einem anderen Blickwinkel folgend lässt sich bemerken, dass wir es auf diesem Felde mit „Konstrukten“ zu tun haben, also nicht mit „Dingen“ oder „Phänomenen“, die man (relativ) direkt messen oder (relativ) umstandslos beobachten könnte, sondern mit Einheiten, die einen konstitutiven Bestandteil haben, der aus einer Vorstellung über ihr Innerstes besteht: Natur, Gesellschaft, Kultur, Technik und Medien verhalten sich so, wie etwa Raum und Zeit, Realität und Virtualität, sowie das Wesen des oder die Identität von Menschen. ${ }^{7}$

Unsere begrifflichen Konzepte der Natur sind - genauso wie unsere Konzepte des Menschen und der Kultur - symbolisch-sprachliche Einheiten („Formen“, „Schemata“), die innerhalb von permanenten gesellschaftlichen Diskursen gebildet und wirksam werden. Als solche stellen sie „soziale Konstruktionen“ dar, deren Bedeutungsinhalte gleichermaßen aufeinander bezogen werden und sich voneinander abheben.

Was „Kultur“ und „Technik“" an sich sind, lässt sich nicht unabhängig davon erörtern, was sie für uns sind, und für die Frage, was ihr Verhältnis ausmacht, gilt das gleiche. Die Bedeutungen, die „Kultur“ und „Technik“ annehmen, die ihr Verhältnis annimmt, sind historisch veränderbare Attributionen, die abhängig von gesellschaftlicher Praxis sind, also von Strukturen, Formen und Mustern des sozialen Wahrnehmens und Handelns. Für das „moderne“ Verständnis von Kultur und Technik spielt dabei nicht zuletzt die Trennung von „Sciences“ und „Humanities“ (bzw. von Natur- und Technik- sowie Geistes- und Sozialwissenschaften) eine entscheidende Rolle, insofern sie ihre begriffliche Distinktion vorprogrammiert. Dies geschieht meistens so, als ob es sich um ontologisch geschiedene Gegenstandsbereiche handeln würde, statt um epistemisch differenzierte Aufmerksamkeitssphären.

„Kultur“ ebenso wie „Sprache“ gibt es eigentlich nirgends, abgesehen von der in den Kulturwissenschaften respektive den Sprachwissenschaften vertretenen Idee, dass alle konkret vorfindbaren, distinkt existierenden Kulturen und natürlichen Sprachen Ausdruck von etwas Essentiellem sind, das den Gegenstand ihrer Wissenschaften bildet. Es lohnt sich also darüber nachzudenken, wie es im Fall der „Technik“ vor dem Hintergrund der Herausbildung der Technikwissenschaften ist.

\footnotetext{
${ }^{7}$ Hinzuweisen ist hier auf die wachsende Bedeutung der sich entwickelnden Beziehungen zwischen „,realen“ und „virtuellen“ Räumen (vgl. u. a. Bühl 2000, S. 156ff.; Castells 2001, S. $431 \mathrm{ff}$.); für Mike Sandbothe stellen „real“ und „virtuell“" „Reflexionsbegriffe“ dar (vgl. Sandbothe 1998, S. 68).
} 
Die distinkte Exklusivität im Verhältnis von Natur- und Kulturwissenschaften spiegelt sich in den Friktionen kulturalistischer und naturalistischer Beschreibungen des Verhältnisses von Umwelt, Technik und Gesellschaft wider. Nicht auszuschließen ist jedoch, dass sich deren Erkenntnisprozesse (vor allem in inter- und transdisziplinären Forschungskooperationen) wechselseitig aufschaukeln können.

\subsection{Vernetzung und Bewegung}

Hinzu tritt allerdings noch etwas, denn alles fließt, es gibt eine Geschichte, eine Entwicklung, und in Phasen in denen Diskontinuitäten und Sprünge auftreten, werden die Begriffe und ihre Bedeutungen unsicher, verflüssigt, offen und sensitiv für neue Zuschreibungen. Sie erfolgen, um etwas bezeichnen und reflektieren zu können, das zwar erkennbar in der Welt geschieht, das aber um so weniger zu begreifen ist, je mehr unsere Begriffe gereinigte und tradierte Bestände darstellen, geschützt von treuhänderisch verfahrenden Disziplinen ${ }^{8}$.

In unserem semantischen Netz oder Bedeutungsgewebe sind alle Begriffe nicht nur miteinander verbunden, sondern verschieben die Inhalte, die sie im Kern ausmachen, wie in einem Kräfteparallelogramm, nämlich durch aufeinander einwirkende wechselseitige Bezugnahmen. Sich wesentlich verändernde Praktiken, auch und gerade insofern sie durch gesellschaftliche Transformationen, technisch-mediale Entwicklungsschübe oder neu sich formierende Problemlagen angetrieben werden, nehmen damit notwendigerweise Einfluss auf das gesamte semantische Netz, nicht nur auf einzelne Begriffe, wie etwa den der „virtuellen“ Identität”.

Klar ist, dass (die) „Kultur“ und (die) „Technik“ (und damit auch das Verhältnis zwischen ihnen) nichts Essentielles sind, sondern ihrerseits etwas gesellschaftlich Konstituiertes, Geformtes und Ausbuchstabiertes sind - etwas sich Entwickelndes sind, Punkte sind, welche sich unter sich verändernden kulturellen, technischen, sozialen oder ökologischen Umgebungsbedingungen verändern, als Phänomene und als Begriffe.

\section{KULTUR \& TECHNIK: EVOLUTIONÄRE GENESE UND EXISTENZIELLE FUNKTIONEN}

3.1 Sozioanthropogenese (ausgehend vom Tier-Mensch-Übergangsfeld)

Der Begriff der „Sozioanthropogenese“ trägt die Bedeutung, dass der evolutionäre Prozess der Menschwerdung und die Herausbildung einer zur sozio-kulturellen Evolution

\footnotetext{
${ }^{8} \mathrm{Ob}$ z. B. eine mit Floureszenz-Genen veränderte Qualle nun ein „Naturwesen“ oder ein „technisches Artefakt“ darstellt, ist nicht nur eine Streitfrage, sondern auch eine erkennbare Belastung für das bisher gültige kategoriale System. Diagnosen wie „Hybride“ (vgl. Latour 1995) oder „Biofakte“ (vgl. Karafyllis 2006) lösen das Problem zwar vordergründig, verschärfen es aber in der Tat, eben weil sie die begriffliche Ambivalenz explizit machen.

${ }^{9}$ Vgl. Metzner-Szigeth, Andreas (2007): Internet \& Gesellschaft: Ein Humanes Projekt? In: Sic et Non. Zeitschrift für Philosophie und Kultur - im Netz, no. 8. - URL: http://www.sicetnon.org/content/pdf/internet\&gesellschaft.pdf.
} 
fähigen Gesellschaft gleichzeitig und abhängig voneinander erfolgten ${ }^{10}$. Jede Tiergesellschaft, mithin jede Affengesellschaft lebt als natürliche Einheit in einer natürlichen Umwelt ${ }^{11}$. Im Übergang zur menschlichen Gesellschaft verschwinden diese beiden Größen nicht, werden aber symbolisch konstituiert: die Einheiten „,menschliche Gesellschaft“ und „ökologische Umwelt" werden so gedoppelt. Sie sind nicht mehr einfach nur eine real existierende Population in einer real existierenden Umwelt, sondern sie sind eine symbolisch existierende (sich selbst beschreibende) Gesellschaft in einer symbolisch existierenden (gesellschaftlich beschriebenen) Umwelt. Menschliche Gesellschaft und ökologische Umwelt „existieren“ also sowohl in einem physischnaturalen, als auch in einem kulturell-symbolischen Sinne ${ }^{12}$.

Die „symbolische Konstituierung der Welt“ erlaubt es, sich ihr gegenüber hypothetisch zu verhalten, und zwar sowohl im Verhältnis zur äußeren, physischen Umwelt, zur sozialen, intermediären Mitwelt, als auch zur inneren, psychischen Innenwelt. Sie ist (evolutionär gesehen) etwas qualitativ Neues, ein Spezifikum der „conditio humana“. Sie eröffnet die Freiheitsgrade, die das menschliche Handeln gegenüber dem tierischen Verhalten auszeichnen. Sie ermöglicht eine Vergegenwärtigung intendierten Handelns innerhalb unserer Vorstellung und entlastet uns von der Notwendigkeit gleichzeitig konkret handeln zu müssen. Durch die symbolische Konstituierung der Erfahrungswelt innerhalb der Sprache wird nicht nur die Möglichkeit geschaffen, eine „Haltung“ gegenüber der äußeren Wirklichkeit einzunehmen, sondern auch gegenüber dem eigenen inneren Erleben, so dass in der Folge das Verhältnis von innerem Erleben und äußerer Welt in einem bis dahin unbekannten Maße verfügbar wird $^{13}$.

Vor diesem Hintergrund ist erkennbar, dass Modelle, die die Wechselwirkungen natürlicher und sozialer Prozesse abbilden wollen, nicht darauf abstellen können, dass es einfache Ursache/Wirkungs-Beziehungen zwischen diesen geben könnte, denn zwischen physischen Ereignissen (oder Bedingungen) und ihren gesellschaftlichen Wirkungen (oder

\footnotetext{
${ }^{10}$ Vgl. Leakey, Richard; Lewin, Roger (1993): Der Ursprung des Menschen. Frankfurt am Main; Löther, Rolf (Hg.) (1988): Tiersozietäten und Menschengesellschaften. Philosophische und evolutionsbiologische Aspekte der Soziogenese. Jena

${ }^{11}$ Vgl. Ploog, Detlev (1972): Kommunikation in Affengesellschaften und deren Bedeutung für die Verständigungsweisen des Menschen. In: Gadamer, Hans-Georg; Vogler, Paul (Hg.): Neue Anthropologie. Bd. 2: Biologische Anthropologie. Zweiter Teil. Stuttgart/München, S. 98-178; Rodseth, Lars; Wrangham, Richard W.; Harrigan, Alisa M.; Smuts, Barbara B. (1991): The Human Community as a Primate Society. In: Current Anthropology, vol. 32, no. 3 (June), pp. 221-254

${ }^{12}$ Vgl. Fischer-Kowalski, Marina; Weisz, Helga (1998): Gesellschaft als Verzahnung materieller und symbolischer Welten. In: Brand, Karl-Werner (Hg.): Soziologie und Natur. Theoretische Perspektiven. Leverkusen-Opladen; Redclift, Michael; Woodgate, Graham (1997): Sustainability and Social Construction. In: Redclift, Michael; Woodgate, Graham (eds.): The International Handbook of Environmental Sociology. Cheltenham, UK/Northampton, US, p. 61

${ }^{13}$ Die symbolische Konstituierung der Welt ist damit die Voraussetzung jeglicher Planung (bzw. aller bewusstseinsgetragenen Entscheidungsprozesse), insofern sie die Möglichkeiten des Durchspielens von Handlungsoptionen im Zusammenhang ihrer Bedingungen und Folgen eröffnet. Sie ist damit auch die Voraussetzung jeglichen Handelns unter Prämissen des Risikos, da das Bewusstsein der Bindung der Handlungsfolgen an vorausgehende Entscheidungen reflexiv verfügbar wird (vgl. Metzner 2002).
} 
Konsequenzen) liegt die Kontingenz menschlichen Wahrnehmens, Entscheidens und Handelns ${ }^{14}$.

Als Moment der Unterscheidung von tierischem Verhalten von menschlichem Handeln $^{15}$ wurde der Begriff der Kontingenz von Max Weber (in Anschluss an verschiedene Anthropologen) in die Gesellschaftswissenschaften eingeführt und durch die Systemtheorie weiter etabliert. Kontingenz meint, dass es für Akteure möglich ist, auf verschiedene Weise zu „handeln“, statt sich bestimmten Bedingungen gegenüber nur auf eine Weise ,verhalten“ zu können. Handeln ist in diesem Sinne nicht determiniert durch eine Kombination feststehender innerer Mechanismen (Verhaltensdispositionen im Sinne der Instinkttheorie oder des Behaviorismus) und externaler (physischer) (Verhaltens)Bedingungen, sondern wird (intrinsisch) determiniert durch symbolisch konstituierte Situationen, Ziele und Mittel, die einen mit Bedeutung aufgeladenen „Bezugsrahmen des Handelns ${ }^{\text {"16 }}$ bilden.

Unabhängig von aller möglichen systemischen Geschlossenheit des Handelns und Kommunizierens sind diese Tätigkeiten keineswegs etwas Selbstzweckhaftes, im Sinne elementarer Operationen, die unabhängig von menschlichen Akteuren und den Funktionen, die sie für diese erfüllen, betrachtet werden könnten. Sie sind an die Existenz von Menschen (und ihre sozialen und ökologischen Existenzbedingungen) gebundene Operationen, die Bedürfnisse und die Möglichkeiten ihrer Befriedigung vermitteln. Diese Bedürfnisse sind die eines lebendigen, selbstbewussten Organismus, dessen Existenz physisch-organisch, psychisch und sozio-kulturell zu verwirklichen ist ${ }^{17}$.

Soziale Systeme zeichnen sich im Allgemeinen gegenüber bloßen Populationen durch Einschränkung der Konkurrenz unter Individuen zugunsten von Kooperation aus. Beim Menschen erfolgt diese Regulierung wesentlich nicht mehr durch biologische Verhaltensprogramme, sondern durch soziale Institutionen, die Aktivitäts-Schemata bereitstellen, die symbolsprachlich sinnprozessierend konstituiert werden. In diesem Sinne sind „soziale Systeme“ als „konstruktive“ Gebilde zu verstehen, die innerhalb von kommunikativen Prozessen hervorgebracht werden, die zwischen menschlichen Akteuren hin- und herlaufen und ihr Handeln orientieren.

\footnotetext{
${ }^{14}$ Vgl. auch Metzner, Andreas (1993): Probleme sozio-ökologischer Systemtheorie - Natur und Gesellschaft in der Soziologie Luhmanns. Opladen. - Reprint: URL: http://sammelpunkt.philo.at:8080/1812, s. 134ff.

${ }^{15}$ Mangels eines besseren Ansatzes verwende ich dieses distinktive Konzept weiter, wohl wissend, dass im Lichte der modernen Primatenforschung nicht mehr länger von einer scharfen Trennung zwischen Tieren und Menschen auszugehen ist. Dies ergibt sich sowohl hinsichtlich der untersuchten Fähigkeiten des Erlernens, des Gebrauchs und der Erzeugung symbolisch-sprachlicher Zeichen, als auch hinsichtlich der untersuchten Fähigkeiten zur Tradierung oder Enkulturation von Techniken des Gebrauchs und der Herstellung von Werkzeugen

${ }^{16}$ Vgl. Parsons, Talcott (1978): Action Theory and the Human Condition. New York/London.

${ }^{17}$ Vgl. Maturana, Humberto R. (1980): Man and Society. In: Benseler, Frank; Hejl, Peter M.; Köck, Wolfram K. (eds.): Autopoiesis, Communication and Society. The Theory of Autopoietic System in the Social Sciences. Frankfurt am Main/New York 1980, pp. 11-31; Maturana, Humberto R. (1987): Biologie der Sozialität. In: Schmidt, Siegfried J. (Hg.): Der Radikale Konstruktivismus. Ein neues Paradigma im interdisziplinären Diskurs. Frankfurt am Main 1987, S. 287-302.
} 


\subsection{Symbolische und materielle Kultur (in der Ökologie der Gesellschaft)}

Selbst unter Berücksichtigung der Relativität der Aussage, dass der Mensch anthropologisch als „Mängelwesen“ zu verstehen sei, liegt die Auffassung nahe, dass die Lebensweise des Menschen existentiell dadurch bestimmt wird, dass er sich eine artifizielle „Welt" schaffen muss, die seine Reproduktion als Gattung innerhalb einer ökologischen Umwelt vermittelt.

Diese ,artifizielle Welt“ ist aber keineswegs einfach mit Kultur im herkömmlich vorherrschenden Sinne gleichzusetzen, denn sie umfasst notwendigerweise nicht nur die symbolsprachlich-kommunikative Kultur, sondern gleichermaßen die materiell-technische Kultur. Sie im Sinne von Arnold Gehlen als „Zweite Natur“ des Menschen zu begreifen ${ }^{18}$, ist also durchaus angemessen. Seine These von der Erfindung sozialer Institutionen zur Absorption der Unsicherheiten des „riskierten Wesens“ trifft allerdings höchstens eine Seite der Medaille ${ }^{19}$. Direkt in den Zusammenhang der Selbstorganisation und Selbstherstellung des Sozialen fällt mit mindestens ebensoviel Gewicht auch die Technologie.

Technisierung ist - kulturanthropologisch gesehen - ein nicht weniger geeignetes Mittel als die Institutionalisierung sozialen Handelns, wenn es um die Absorption von Unsicherheiten geht ${ }^{20}$. Sie stellt einen ebenso sozialen wie materiellen Prozess dar. Auch sie verändert Bedingungen des Handelns durch Handeln.

Technik und Sprache lassen sich - der Abhandlung über „Hand und Wort“ von André Leroi-Gourhan folgend - als gleichermaßen wichtige Medien der menschlichen Vergesellschaftung begreifen, mit ebenso großer Relevanz für die Ausdifferenzierung sozialer Systeme.

Als Katalysatoren von Vergesellschaftung fungieren also nicht nur normative, sondern auch stoffliche Strukturen, nicht nur symbolische Kommunikationsmedien, sondern auch technische Infrastruktursysteme, um die herum sich geordnete Systeme des Handelns verdichten.

Als Hervorbringung der menschlichen Gesellschaft stellt Kultur keinen Selbstzweck dar, sondern sie ist notwendige und permanente Bedingung der Existenz und Erhaltung jeder menschlichen Lebensweise. Erst sie ermöglicht das menschliche Leben, indem sie seine Auseinandersetzung mit der ökologischen Umwelt ganzheitlich vermittelt und organisiert. Eine Leistung der sozialen, kommunikativen Kultur ist hierbei die Konstruktion einer gesellschaftlichen Wirklichkeit, ist das Konstruieren einer Gesellschaft und Natur umfassenden differenzierten und komplexen „Welt“ (oder Weltsicht), anhand derer sich Menschen in ihrer Umwelt in ihrem Handeln orientieren ${ }^{21}$. Eine Leistung der materiellen, technischen Kultur ist das tätige, kreative Hervorbringen, das Produzieren

\footnotetext{
${ }^{18}$ Vgl. Gehlen, Arnold (1978): Der Mensch. Seine Natur und seine Stellung in der Welt [1940]. 12. Aufl. Wiesbaden, s. 38, 80f.

19 Vgl. Claessens, Dieter (1980): Das Konkrete und das Abstrakte. Soziologische Skizzen zur Anthropologie. Frankfurt am Main; Schülein, Johann August (1987): Theorie der Institution. Eine dogmengeschichtliche und konzeptionelle Analyse. Opladen.

${ }^{20}$ Vgl. Halfmann, Jost (1996): Die gesellschaftliche „Natur“ der Technik. Eine Einführung in die soziologische Theorie der Technik. Leverkusen-Opladen, s. 91ff., insb. 94ff.; vgl. hierzu auch Grunwald in diesem Band.

${ }^{21}$ Vgl. Berger, Peter L.; Luckmann, Thomas (1966): The Social Construction of Reality. A Treatise in the Sociology of Knowledge. New York.
} 
einer artifiziellen (Um-) Welt von - auch symbolisch besetzten - Gegenständen, also Behausungen, Werkzeugen, Werk- und Wirkstoffen sowie „Lebens-Mitteln“ aller Art ${ }^{22}$. Beide Aspekte menschlicher Kultur erfüllen so gesehen zwar existentielle Funktionen, sind aber trotzdem nicht einfach instrumentell als Werkzeuge des „Überlebens“ oder gar der „Anpassung“ zu verstehen, denn aus Kultur kann man nicht aussteigen, sie ist systemisch, umgibt den Menschen, indem sie ihn erschafft. Kultur ist daher beides zugleich, Zweck und Mittel. Als menschliche Kultur ermöglicht sich das menschliche Leben selbst und besteht innerhalb dieser systemischen Rekursion fort: Menschen „leben“ dann nicht nur in von ihnen symbolisch erschlossenen, sondern gleichermaßen in von ihnen tätig veränderten „Welten“23.

\subsubsection{Basis und Überbau?}

Um Verlusten vorzubeugen, ist hier darauf hinzuweisen, dass unabhängig von der Unstimmigkeit anderer, wichtiger Elemente seines Theoriegebäudes, es auch heute noch als Verdienst von Karl Marx gelten darf, die notwendige, durch „lebendige Arbeit“ vermittelte, dialektische Verbindung der materiellen („ökonomischen“) Reproduktion und der ideellen (,politischen“) Gestaltung von Gesellschaft hervorgehoben zu haben. $\mathrm{Ob}$ erstere der letzteren im Sinne des Basis/Überbau-Modells vorgeordnet werden kann, ist aber stark zu bezweifeln.

Folgt man statt dessen den Überlegungen des Kulturanthropologen Marshall D. Sahlins, so lässt sich mit ihm erstens einsehen, „daß sich die materiellen Aspekte nicht sinnvoll von den gesellschaftlichen trennen lassen, so als ob erstere sich auf die Bedürfnisbefriedigung durch die Ausbeutung der Natur bezögen und letztere auf die Probleme der Beziehungen zwischen den Menschen““24 ${ }^{25}$. Zweitens lässt sich mit ihm Folgendes begreifen: „Sowohl die Zweckbestimmungen als auch die Art und Weise, wie produziert wird, hängen von der Kultur ab: die materiellen Mittel der kulturellen Organisation wie auch die Organisation der materiellen Mittel“‘26 ${ }^{27}$

\subsubsection{Prozesse der Anpassung und des Lernens}

Ohne seine Kulturfähigkeit ist die ubiquitäre Verbreitung des Menschen, der in allen Klimazonen siedelt, nicht zu erklären. Sie stellt den entscheidenden Faktor dar, denn unter

\footnotetext{
${ }^{22}$ Vgl. Leroi-Gourhan, André (1973): Milieu et technique. Paris; Tinland, Frank (1977): La différence anthropologique. Essai sur les rapports de la nature et de l'artifice. Paris.

${ }^{23}$ Vgl. Morin, Edgar (1973): Le paradigme perdu: la nature humaine. Paris.

${ }^{24}$ Sahlins, Marshall D. (1981): Kultur und praktische Vernunft. Frankfurt am Main, s. 288.

${ }^{25}$ Eine solche Trennung müsste dazu führen, entweder deterministisch das Soziale aus dem Materiellen ableiten zu wollen, oder relativistisch zu verfahren: Die materielle und die symbolische Kultur stehen dann als isolierte nebeneinander, wobei für die eine der Schein der Naturnotwendigkeit, für die andere der der Voraussetzungslosigkeit entsteht. Sahlins betrachtet hingegen sowohl die technische wie die nicht-technische Kultur als symbolisch konstituiert auf der Basis geteilter, fundamentaler Bedeutungssysteme.

${ }^{26}$ Sahlins, Marshall D. (1981): Kultur und praktische Vernunft. Frankfurt am Main, s. 291.

${ }^{27}$ Um die soziale „Aneignung“ der Natur zu verstehen, gilt es folglich zu rekapitulieren, wie diese in ihrer innigen Verbindung ihrer kulturell-kommunikativen und physisch-materiellen Momente gelingt (vgl. auch Eder 1988)
} 
Rekurs auf biologische Anpassung ist dieser Befund nicht plausibel zu machen ${ }^{28}$. Im Vergleich zum Verhaltensrepertoire anderer Spezies generiert Kultur eine Adaptibilität besonderer Art. Mittel derselben sind die Herausbildung verschiedener Strategien der Nahrungsgewinnung, distinkter Strukturen des Zusammenlebens, unterschiedlicher Mechanismen der direkten und indirekten Bevölkerungsregulation, variierender Muster der Herstellung und Nutzung von Technologien etc., die „angepasst“, das heißt in bestimmten Umwelten effektiv sind. Als Anpassungsvorgänge an die ökologischen Ressourcen und Bedingungen verschiedenster Biotope und Klimazonen sind diese kulturellen Phänomene insofern von „Anpassung“ im Sinne der organischen Evolution zu unterscheiden, als sie nicht genetisch, durch zufällige Variationen und Selektion erfolgen, sondern durch Erfahrung, Lernen, Reflexion und Tradierung. Die menschliche Befähigung zur ,aktiven (zielgerichteten, intentionalen) Anpassung“ an externe Bedingungen beruht auf sozio-kulturellen Lernprozessen, die dafür verantwortlich sind, dass er einzigartige Fähigkeiten zur „aktiven Umgestaltung“ seiner Umwelt aufbauen konnte $^{29}$.

Die unterschiedlichen Eigenschaften ökologischer Räume (geographische Lage, Nahrungs- wie Wasserangebot, Ressourcen, Klima etc.) sowie ihre Art und Weise der Interaktion mit der physischen Umwelt (Technologien, Kooperationsformen etc.) beeinflussen das Bild, das sich die Menschen in einer gegebenen Kultur von der sie umgebenden Natur, von sich selbst, und von ihrer Beziehung zur Umwelt machen. Dieses Bild ist präsent in ihren Vorstellungen, ihrem Denken und Fühlen. Es gewinnt Bedeutung in ihren Normen, Werten und Verhaltensweisen, aber auch in ihren Technologien. Das heißt: sowohl die physisch-materielle Kultur einer Gesellschaft (ausgedrückt in ihren Werkzeugen, Gebrauchsgegenständen, Technologien und ihrer baulichen Infrastruktur), als auch die symbolisch-kommunikative Kultur einer Gesellschaft (ausgedrückt in Werten und Normen, Mythen und Weltanschauungen) ist durchtränkt mit der kulturell gebildeten Bedeutung der Natur für den Menschen, die sich im Kontext seiner Auseinandersetzung mit der ihn umgebenden Natur herauskristallisiert und verändert ${ }^{30}$.

\subsection{Zur Konstituierung gesellschaftlicher Wirklichkeit}

Die Konstituierung gesellschaftlicher Wirklichkeit lässt sich - die bisherigen Überlegungen im Sinn - als ein dreifach abgestufter Prozess begreifen: Gesellschaften verändern die physische Wirklichkeit durch die tätige Verarbeitung von Stoffen in Kombination mit der Umwandlung von Energien, die psychische Wirklichkeit durch die Konstruktion der Bedeutung von Wahrnehmungsinhalten und Handlungsmotiven innerhalb von Kommunikationsprozessen und ordnen ihre eigene soziale Wirklichkeit durch Institutionen, die beides vermitteln.

\footnotetext{
${ }^{28}$ Vgl. Campbell, Bernard (1987): Ökologie des Menschen. Unsere Stellung in der Natur von der Vorzeit bis heute. Frankfurt am Main/Berlin.

${ }^{29}$ Vgl. Casimir, Michael J. (1993): Gegenstandsbereiche der Kulturökologie. In: Schweizer, Thomas; Schweizer, Margarete; Kokot, Waltraud (Hg.): Handbuch der Ethnologie. Berlin, S. 217ff.

${ }^{30}$ Vgl. Casimir, Michael J. (1993): Gegenstandsbereiche der Kulturökologie. In: Schweizer, Thomas; Schweizer, Margarete; Kokot, Waltraud (Hg.): Handbuch der Ethnologie. Berlin, S. 222; Kaplan, Stephen; Kaplan, Rachel (1981): Cognition and Environment. Functioning in an Uncertain World. New York.
} 
Unterscheidet man - vor dem Hintergrund des symbolischen Interaktionismus von George Herbert Mead $^{31}$ - mit Peter L. Berger und Thomas Luckmann Externalisierung, Objektivation und Internalisierung als drei Momente der dialektischen Vermittlung zwischen Akteuren und der von ihnen hervorgebrachten Welt ${ }^{32}$, so lässt sich - und das ist die hier vertretene These - dieser Dreisatz nicht nur wissenssoziologisch auf die symbolische Hervorbringung von sozialer Wirklichkeit anwenden, sondern auch auf die materielle.

Klaus P. Japp schreibt hierzu: „Institutionentheorien gehen davon aus, daß die aggregierten Effekte von Handlungen sich diesen gegenüber verselbständigen und den Charakter einer ,objektiven Realität' annehmen“"33. Genau in diesem Sinne vertritt die hier entwickelte These die Auffassung, dass nicht nur Institutionen, sondern auch Technologien den sozial Handelnden, die sie hervorbringen, als zeiträumlich fixierte (,objektive“) Handlungsbedingungen gegenübertreten. Damit sind begründungsentlastete (oder wenn man so will: von doppelter Kontingenz entlastete) Prozesse der Herausbildung und Stabilisierung von (gängigen) Handlungsweisen und Handlungsmustern ermöglicht und das bedeutet nichts anderes als ein Vergesellschaftungsprinzip!

Bauliche und technische Artefakte, infrastrukturelle Systeme, aber auch Werk- und Wirkstoffe aller Art sind Produkte menschlicher Entäußerung. Sie vergegenständlichen sich zu einer dinglichen Welt, die sich dem Wollen der Subjekte ein Stück weit entzieht und sie wird von diesen als (zeiträumlich) fixierte Handlungsumgebung mitsamt der dadurch gegebenen Möglichkeiten und Beschränkungen internalisiert.

In diesem Zusammenhang liegt auch die Möglichkeit begründet, dass sich die dingliche Welt des technischen Fortschritts als von Menschen unkontrolliertes Risiko, als Gefahr, darstellt, aber auch die Möglichkeit, dass sich der Lauf des Fortschritts als eine autonome Macht darstellt, unabhängig davon, ob sie als „Fluch“ oder „Segen“ wahrgenommen wird. Wendet man diesen (dialektischen) Zusammenhang reflexiv, ergibt sich, dass gesellschaftliche Gestaltungsprozesse symbolische und dingliche Dimensionen auf eine bestimmte Weise miteinander verbinden und dass genau diese Schnittstelle aktiv veränderbar ist.

\section{KULTUR \& TECHNIK: ALS ZWECKE, MITTEL UND MEDIEN}

\subsection{Zur Doppelgestalt des Menschen als Natur- und Kulturwesen}

Betrachten wir den Menschen als Zugleich von Natur- und Kulturwesen, so ließe sich die begriffliche Differenz von bestimmbar und unbestimmbar hieran ansetzend entwickeln: als Naturwesen ist der Mensch (objektivierend) bestimmbar, als Kulturwesen nicht. Als Kulturwesen bestimmt der Mensch sich selber!

\footnotetext{
31 Vgl. Mead, George Herbert (1988): Geist, Identität und Gesellschaft aus der Sicht der Sozialbehavioris-mus. 7. Aufl. Frankfurt am Main

${ }^{32}$ Vgl. Berger, Peter L.; Luckmann, Thomas (1966): The Social Construction of Reality. A Treatise in the Sociology of Knowledge. New York.

33 Japp, Klaus P. (1996): Soziologische Risikotheorie. Funktionale Differenzierung, Politisierung und Reflexion. Weinheim/München, s. 35.
} 
Allerdings ist diese Überlegung so nicht abzuschließen. Als individuelles Wesen ist jeder Mensch in hohem Maße bestimmt, durch die besondere Kultur, in die er hineinwächst (,Sozialisation“).

Kultur wiederum entsteht in Prozessen kognitiver, emotionaler und praktischer Auseinandersetzung von interagierenden Individuen mit den Bedingungen ihres Handelns, die ihrerseits nicht nur soziale, sondern auch technische und ökologische Dimensionen umfassen.

Als Vorgaben für gesellschaftliche Prozesse sind beide, als je aktueller Stand weiter veränder- und entwickelbar, aber nicht in beliebiger Weise, denn auch als der menschlichen Gestaltung offen stehende Phänomene folgen Technik und Ökologie eigenen Gesetzmäßigkeiten, die qua Natur bestimmt werden und sich von den Gesetzmäßigkeiten unterscheiden, die für soziale und kulturelle Systeme (im engeren Sinne) gelten.

Damit liegt die These nahe, dass der Mensch als Kulturwesen weder einfach „unbestimmt“, noch einfach „selbstbestimmt“ ist. Statt dessen ist folgende Marx'sche These nach wie vor aktuell: „Die Menschen machen ihre eigene Geschichte, aber sie machen sie nicht aus freien Stücken, nicht unter selbstgewählten, sondern unter unmittelbar vorgefundenen, gegebenen und überlieferten Umständen““34.

Überhaupt ein Verhältnis von Natur und Kultur zu haben, ist das, was die menschliche Gesellschaft als solche auszeichnet ${ }^{35}$. Die Gestalten, Inhalte und Ausprägungen dieses Verhältnisses kennzeichnen jede besondere Gesellschaft. Wenn es also etwas gibt, was von fundamentaler Bedeutung für die Leitbilder zur Gestaltung unserer Zukunft ist, dann liegt es in diesem Verhältnis verborgen.

Warum sollte also - um auf ein paar aktuelle Debatten anzuspielen - für die Wissenskultur der Nachhaltigkeit, die Gestaltung der elektronischen Räume und den Aufbau der „knowledge-based society“ etwas anderes gelten, als für die menschliche Kultur überhaupt? Sie muss sich bewähren, gegenüber der äußeren und der inneren Natur und zwar im Angesicht der Aufgaben, vor die sie sich selbst stellt. Kultur ist daher weniger ein passives Rezipieren (geschweige denn ein bloßes Konsumieren) von Kulturgütern, sondern vielmehr ein aktives Hervorbringen. Auf den Punkt bringt dies ein „Bonmot“ von Antoine de Saint-Exupéry: „Eine Kultur beruht auf dem, was von den Menschen gefordert wird, und nicht auf dem, was sie geliefert erhalten“.

Insofern das für die Entwicklung des menschlichen Lebens (von Beginn an und nach wie vor) existenzielle Verhältnis von der Gesellschaft gegenüber und mit der Natur durch die tätigen, handelnden Hervorbringungen von Kultur und Technik vermittelt, formiert und gestaltet wird, lassen sich diese - so die hier vertretene These - als (gleichursprüngliche aber seinsverschiedene) Medien der (Selbst-)Verwirklichung des Menschen als Menschen bestimmen.

\footnotetext{
${ }^{34}$ Marx, Karl (1960): Der 18te Brumaire des Louis Napoleon [1852]. In: Marx, Karl; Engels, Friedrich: Werke. Bd. 8. Berlin, S. 115.

${ }_{35}$ Neben der philosophischen Begründung dieser These (vgl. u. a. Moscovici 1968) liegt die Möglichkeit ihrer evolutionstheoretischen Herleitung (vgl. u. a. Harris 1979, 1989); beide Stränge fließen in den Denkzusammenhang einer (weit gefassten) „Kulturökologie“ ein (vgl. u. a. Bargatzky 1986; Glaeser/Teherani-Krönner 1992), die sie auch vergleichend diskutieren könnte.
} 


\subsection{Exkurs zum Begriff des Mediums}

Das „Medium“ konnotiert, Marshall McLuhans’ Diktum „the medium is the message“ folgend $^{36}$, mit einem Verständnis, in dem es keineswegs nur den Charakter eines Mittels (für etwas) trägt, sondern auch den eines Zweckes (an sich).

Medien dienen der Speicherung und Wiedergabe von Information sowie der Übertragung und Vermittlung von Kommunikation, wobei unter „Kommunizieren“ üblicherweise ein Austausch zwischen Individuen verstanden wird, der auf Gegenseitigkeit und Wechselwirkung basiert, während „Informieren“ als Prozess gilt, der nur in eine Richtung erfolgt. Beide Vorgänge lassen sich dabei nicht auf den physikalischen Vorgang des „Sendens und Empfangens“ reduzieren, weil sie notwendigerweise auch als Bedeutungen „enkodierende“ und „dekodierende“ interpretative Akte in einem sozialen „Umfeld“ und vor einem kulturellen „Hintergrund“ begriffen werden müssen ${ }^{37}$. Ihr Begriff ist daher von vorne herein darauf angelegt, Systeme der Informations- und Kommunikationsvermittlung sowohl nach ihrer technischen als auch nach ihrer kulturellen Seite hin zu erschließen, wobei quer dazu einerseits ihre „Funktion“ hinsichtlich des „Transports“ von Inhalten im Vordergrund steht und zum anderen die „Form“, in der dies geschieht und dabei die Möglichkeiten der „Verständigung“ mit Anderen und der „Erfahrung“ der Welt prägt.

Infolge von Wissenschaftsdualismus und professioneller Arbeitsteilung werden Medien üblicherweise entweder als (eher) artifiziell-technische Systeme oder als (eher) sozio-kulturelle Praktiken verstanden. Einer ganzheitlichen Medientheorie muss es jedoch darauf ankommen, beide Seiten in ihrer Verbindung miteinander zu erschließen, denn gerade in ihren Wechselwirkungen bildet sich das Spezifische eines jeden einzelnen, besonderen Mediums heraus.

Im Weiteren anzusprechen sind hier zwei zu einseitige Konzeptualisierungen. In Bezug auf die (neuen) Medien lässt sich zwischen einer (eher) medientheoretischen und einer (eher) kommunikationstheoretischen Engführung unterscheiden ${ }^{38}$.

Die medientheoretische Engführung beruht auf der Denkfigur, dass das Medium selbst unabhängig von seiner Verwendung das Verhältnis des Menschen zur Welt verändert. Die Übertreibung dieser Denkfigur lautet: jedes Medium schafft eine andere Weltsicht. In dieser Übertreibung liegt ein stark vereinfachtes Verständnis über den Zusammenhang von Medium und Kommunikation, dass nämlich jede neue Medientechnologie eine andere Kommunikationsweise durchsetzt und die alten Formen der Kommunikation ablöst.

Die kommunikationstheoretische Engführung resultiert aus der Übertreibung der Denkfigur, dass das Medium ein neutrales Mittel sei, das neue Möglichkeiten eröffne, und dass es allein auf die menschlichen Akteure und ihre Handlungen ankomme, wie sich durch Mediennutzung die Kommunikations- und Interaktionsverhältnisse verändern. Diese Denkfigur setzt das Wirkungsspektrum von Medien dem von Werkzeugen gleich.

\footnotetext{
${ }^{36}$ Vgl. McLuhan, Marshall (1964): Understanding Media: The Extensions of Man. Cambridge, MA (dt.: Die magischen Kanäle. Understanding Media. Düsseldorf 1992)

${ }^{37}$ Vgl. Faßler, Manfred; Halbach, Wulf R. (Hg.) (1998): Geschichte der Medien. München.

38 Vgl. Rammert, Werner (1999): Virtuelle Realitäten als medial erzeugte Sonderwirklichkeiten Veränderungen der Kommunikation im Netz der Computer. In: Faßler, Manfred (Hg.): Alle möglichen Welten. München, S. 33-48
} 


\subsection{Rekursion \& Dialektik}

In den beiden zentralen Dimensionen menschlichen Lebens, Kultur und Technik, verweisen die je verschiedenen Möglichkeiten ihrer begrifflichen Erschließung zusammengenommen auf eine Beziehung der Rekursion oder Dialektik zwischen dem Hervorgebrachten und dem etwas Hervorbringenden, zwischen Objekt und Subjekt.

„Kultur" und „Technik“ sind auch - neben anderen Funktionen - als Medien des SichSelbst-Verstehens, des Sich-Selbst-Erkennens zu betrachten ${ }^{39}$, insofern sie auch dazu dienen Bilder und Vorstellungen zu entwickeln, die nicht nur das Moment deskriptiver (Selbst- und Verhältnis-) Beschreibungen enthalten, sondern auch das Moment präskriptiver Vorschriften (Handlungskonzepte, Strategieansätze).

„Kultur“ und „Technik“, als notwendige „Medien“ der Selbstverwirklichung des Menschen als Menschen, verwirklichen ihre Funktion im Verlauf von kontingenten Entwicklungswegen, auf denen sie - als „Projekte“ - miteinander verbundene Linien der Kulturentwicklung (Kulturtechniken) und Technisierung (Technikkulturen) erzeugen.

Darüber hinaus ist noch etwas zu berücksichtigen. Zwar wird die „Substanz“ des Sozialen in gängigen Gesellschaftstheorien je anders bestimmt, Kollektivbewusstsein bei Ėmile Durkheim, Arbeit bei Karl Marx, Geselligkeit bei Georg Simmel, soziales Handeln bei Max Weber, symbolische Interaktion bei Georg H. Mead, soziale Handlungssysteme bei Talcott Parsons, kommunikatives Handeln bei Jürgen Habermas und Kommunikation bei Niklas Luhmann; nichtsdestoweniger gilt für alle das „Dogma“, dass Soziales nur durch Soziales erklärt werden kann. Ohne daran rütteln zu wollen, bleibt zu fragen, inwieweit üblicherweise als nicht soziale Tatsachen gehandelte technische oder ökologische Faktoren „substanziell“ soziale sind, weil sie als Bedingungen und Resultate Momente materialen sozialen Handelns darstellen. In Verbindung mit diesem Problem steht ein Gesichtspunkt, der wichtig ist, um einen übergeordneten (theoretischen) Rahmen aufspannen zu können. Er wird von Werner Rammert identifiziert, der auf eine Neuerung hinweist, die im Schnittfeld von Mensch-Computer-Interaktivität und von zwischenmenschlichen computervermittelten Interaktionen durch die Vernetzung entstanden ist: „So wie die Begegnung zweier Subjekte einen Raum der Intersubjektivität, den wir üblicherweise als Gesellschaft bezeichnen, schaffen, so scheinen die von mehreren Subjekten ausgelösten Interaktivitäten von Programmen und anderen Objekten einen Raum der Interobjektivität und der Intertextualität zu erzeugen, bei dem wir uns noch schwer tun, ihn auch als Teil der Gesellschaft zu erkennen und zu akzeptieren'“40.

Gesellschaft findet so - wie ausgeführt - keineswegs nur im „Medium“ der Intersubjektivität statt, sondern auch in dem der „Interobjektivität“. Das, was bestimmt, ist also nicht hinreichend erfasst, wenn man sagt, es handele sich um das (menschliche, erkennende, wollende) Subjekt; auch nicht, wenn man die interaktiven Netzwerke an Subjekten ins Spiel bringt oder darüber hinaus die eigene Rolle sozialer Systeme betont. Bestimmend ist vielmehr das Netzwerk der intersubjektiven und interobjektiven

\footnotetext{
${ }^{39}$ Vgl. Weizenbaum, Joseph (1977): Die Macht der Computer und die Ohnmacht der Vernunft. Frankfurt am Main, s. 35.

${ }^{40}$ Rammert, Werner (1999): Virtuelle Realitäten als medial erzeugte Sonderwirklichkeiten Veränderungen der Kommunikation im Netz der Computer. In: Faßler, Manfred (Hg.): Alle möglichen Welten. München, S. 33-48
} 
Zusammenhänge, in denen wechselseitige Bestimmungen erfolgen, die das Verhältnis zwischen Subjekt(en) und Objekt(en) gestalten und damit natürlich auch das, was diese einzeln gesehen ausmacht.

„Menschwerdung“ ist so gesehen mehr, als mit Mitteln der Kultur Beschreibungen seiner selbst anzufertigen und zu beginnen, sich diesen Beschreibungen entsprechend (kultiviert, zivilisiert) zu verhalten, sondern ist darüber hinaus ein Prozess des Sich-selbstzugleich-mit-seiner-Welt-Veränderns, der (gleichursprünglich) auf die Mittel der Technik angewiesen ist.

\section{LITERATUR}

[1] Banse, Gerhard (Hg.) (1997): Allgemeine Technologie zwischen Aufklärung und Metatheorie. Johann Beckmann und die Folgen. Berlin

[2] Bargatzky, Thomas (1986): Einführung in die Kulturökologie. Umwelt, Kultur und Gesellschaft. Berlin

[3] Berger, Peter L.; Luckmann, Thomas (1966): The Social Construction of Reality. A Treatise in the Sociology of Knowledge. New York

[4] Böhme, Gernot (1992): Natürlich Natur. Über Natur im Zeitalter ihrer technischen Reproduzierbarkeit. Frankfurt am Main

[5] Bühl, Achim (2000): Die Virtuelle Gesellschaft des 21. Jahrhunderts. Sozialer Wandel im Digitalen Zeitalter. Opladen

[6] Campbell, Bernard (1987): Ökologie des Menschen. Unsere Stellung in der Natur von der Vorzeit bis heute. Frankfurt am Main/Berlin

[7] Casimir, Michael J. (1993): Gegenstandsbereiche der Kulturökologie. In: Schweizer, Thomas; Schweizer, Margarete; Kokot, Waltraud (Hg.): Handbuch der Ethnologie. Berlin, S. 215-239

[8] Castells, Manuel (2001): Das Informationszeitalter - Wirtschaft, Gesellschaft. Kultur. Teil 1: Der Aufstieg der Netzwerkgesellschaft. Opladen

[9] Claessens, Dieter (1980): Das Konkrete und das Abstrakte. Soziologische Skizzen zur Anthropologie. Frankfurt am Main

[10]Eder, Klaus (1988): Die Vergesellschaftung der Natur. Studien zur sozialen Evolution der praktischen Vernunft. Frankfurt am Main

[11]Faßler, Manfred; Halbach, Wulf R. (Hg.) (1998): Geschichte der Medien. München

[12]Fischer-Kowalski, Marina; Weisz, Helga (1998): Gesellschaft als Verzahnung materieller und symbolischer Welten. In: Brand, Karl-Werner (Hg.): Soziologie und Natur. Theoretische Perspektiven. Leverkusen-Opladen, S. 145-172

[13]Gedö, Andras (1986): Die Geschichtlichkeit des Naturbegriffs. In: Dialektik, Bd. 12, S. 99-114

[14]Gehlen, Arnold (1978): Der Mensch. Seine Natur und seine Stellung in der Welt [1940]. 12. Aufl. Wiesbaden

[15]Glaeser, Bernhard; Teherani-Krönner, Parto (Hg.) (1992): Humanökologie und Kulturökologie. Opladen

[16]Habermas, Jürgen (1976): Zur Rekonstruktion des Historischen Materialismus. Frankfurt am Main

[17]Halfmann, Jost (1996): Die gesellschaftliche „Natur“ der Technik. Eine Einführung in die soziologische Theorie der Technik. Leverkusen-Opladen 
[18]Harris, Marvin (1979): Cultural Materialism. The Struggle for a Science of Culture. New York

[19]Harris, Marvin (1989): Kulturanthropologie. Frankfurt am Main

[20]Hörning, Karl Heinz (1985): Technik und Symbol - Ein Beitrag zur Soziologie alltäglichen Technikumgangs. In: Soziale Welt, H. 2, S. 185-207

[21]Japp, Klaus P. (1996): Soziologische Risikotheorie. Funktionale Differenzierung, Politisierung und Reflexion. Weinheim/München

[22]Kaplan, Stephen; Kaplan, Rachel (1981): Cognition and Environment. Functioning in an Uncertain World. New York

[23] Karafyllis, Nicole C. (2006): Biofakte - Grundlagen, Probleme, Perspektiven. In: Erwägen Wissen Ethik, Jg. 17, H. 4, S. 547-558

[24]Keil-Slawik, Reinhard (2003): Technik als Denkzeug. Lerngewebe und Bildungsinfrastrukturen. In: Keil-Slawik, Reinhard; Kerres, Michael (Hg.): Wirkungen und Wirksamkeit neuer Medien in der Bildung. Münster, S. 13-29

[25]Laitko, Hubert (1986): Natur - Zur Entwicklung einer philosophischen und wissenschaftlichen Kategorie. In: Dialektik, Bd. 12, S. 115-130

[26]Latour, Bruno (1995): Wir sind nie modern gewesen. Berlin

[27]Leakey, Richard; Lewin, Roger (1993): Der Ursprung des Menschen. Frankfurt am Main

[28]Leroi-Gourhan, André (1973): Milieu et technique. Paris

[29]Leroi-Gourhan, André (1988): Hand und Wort. Über die Evolution von Technik, Sprache und Kunst. Frankfurt am Main

[30]Löther, Rolf (Hg.) (1988): Tiersozietäten und Menschengesellschaften. Philosophische und evolutionsbiologische Aspekte der Soziogenese. Jena

[31]Marx, Karl (1960): Der 18te Brumaire des Louis Napoleon [1852]. In: Marx, Karl; Engels, Friedrich: Werke. Bd. 8. Berlin, S. 113-207

[32]Maturana, Humberto R. (1987): Biologie der Sozialität. In: Schmidt, Siegfried J. (Hg.): Der Radikale Konstruktivismus. Ein neues Paradigma im interdisziplinären Diskurs. Frankfurt am Main 1987, S. 287-302

[33]Maturana, Humberto R. (1980): Man and Society. In: Benseler, Frank; Hejl, Peter M.; Köck, Wolfram K. (eds.): Autopoiesis, Communication and Society. The Theory of Autopoietic System in the Social Sciences. Frankfurt am Main/New York 1980, pp. 11-31

[34]McLuhan, Marshall (1964): Understanding Media: The Extensions of Man. Cambridge, MA (dt.: Die magischen Kanäle. Understanding Media. Düsseldorf 1992)

[35]Mead, George Herbert (1988): Geist, Identität und Gesellschaft aus der Sicht der Sozialbehavioris-mus. 7. Aufl. Frankfurt am Main

[36]Metzner, Andreas (1993): Probleme sozio-ökologischer Systemtheorie - Natur und Gesellschaft in der Soziologie Luhmanns. Opladen. - Reprint: URL: http://sammelpunkt.philo.at:8080/1812

[37]Metzner, Andreas (2002): Die Tücken der Objekte. Über die Risiken der Gesellschaft und ihre Wirklichkeit. Frankfurt am Main

[38]Metzner-Szigeth, Andreas (2007): Internet \& Gesellschaft: Ein Humanes Projekt? In: Sic et Non. Zeitschrift für Philosophie und Kultur - im Netz, no. 8. URL: http://www.sicetnon.org/content/pdf/internet\&gesellschaft.pdf

[39]Morin, Edgar (1973): Le paradigme perdu: la nature humaine. Paris 
[40]Moscovici, Serge (1968): Essai sur l'histoire humaine de la nature. Paris (dt.: Versuch über die menschliche Geschichte der Natur. Frankfurt am Main 1982)

[41]Parsons, Talcott (1978): Action Theory and the Human Condition. New York/London

[42]Ploog, Detlev (1972): Kommunikation in Affengesellschaften und deren Bedeutung für die Verständigungsweisen des Menschen. In: Gadamer, HansGeorg; Vogler, Paul (Hg.): Neue Anthropologie. Bd. 2: Biologische Anthropologie. Zweiter Teil. Stuttgart/München, S. 98-178

[43]Rammert, Werner (1999): Virtuelle Realitäten als medial erzeugte Sonderwirklichkeiten - Veränderungen der Kommunikation im Netz der Computer. In: Faßler, Manfred (Hg.): Alle möglichen Welten. München, S. 3348

[44]Redclift, Michael; Woodgate, Graham (1997): Sustainability and Social Construction. In: Redclift, Michael; Woodgate, Graham (eds.): The International Handbook of Environmental Sociology. Cheltenham, UK/Northampton, US, pp. $55-82$

[45]Rodseth, Lars; Wrangham, Richard W.; Harrigan, Alisa M.; Smuts, Barbara B. (1991): The Human Community as a Primate Society. In: Current Anthropology, vol. 32 , no. 3 (June), pp. 221-254

[46]Ropohl, Günter (1993): Technik. In: Brockhaus-Enzyklopädie. Bd. 21. Mannheim, S. 672-674

[47]Sahlins, Marshall D. (1981): Kultur und praktische Vernunft. Frankfurt am Main

[48]Sandbothe, Mike (1998): Transversale Medienwelten. Philosophische Überlegungen zum Internet. In: Vattimo, Gianni; Welsch, Wolfgang (Hg.): Medien, Welten, Wirklichkeiten. München, S. 59-83

[49]Schülein, Johann August (1987): Theorie der Institution. Eine dogmengeschichtliche und konzeptionelle Analyse. Opladen

[50]Tinland, Frank (1977): La différence anthropologique. Essai sur les rapports de la nature et de l'artifice. Paris

[51] Weizenbaum, Joseph (1977): Die Macht der Computer und die Ohnmacht der Vernunft. Frankfurt am Main

\section{TECHNIKA I KULTURA JAKO MEDIA ZRÓWNOWAŻONEGO ROZWOJU - CZY ISTNIEJE NEXUS MIEDZY EKOLOGIA CZŁOWIEKA, ANTROPOLOGIĄ FILOZOFICZNA A TEORIĄ SPOLECZNĄ?}

Pełnia strategii tematyzacyjnych i oramowań refleksyjnych, ukierunkowanych na identyfikację stosunku między kulturą a techniką, odzwierciedla złożoność wzajemnych uwarunkowań i wpływów, z jakimi oba fenomeny na siebie oddziaływają. Niezależnie od tego, na jaki obszar może się rozciągać identyfikacja wzajemnych związków kultury i techniki, należy zakładać, że wzajemne zależności teorii i praktyki, które stanowią jej podstawę, umożliwiają sensowny dyskurs między wszystkimi koncepcjami, niezależnie od tego, z jakiej pozycji, w jaki sposób i przy pomocy jakich środków dane koncepcje identyfikuja ten stosunek. Dlatego jest pewne, że nie tylko konieczne, ale i możliwe jest koncentrowanie uwagi na określonych wycinkach lub charakterystycznych połączeniach, nie narażające rozważań na płaszczyźnie substancjalnej na niebezpieczeństwo nieznalezienia żadnego istotowego dostępu do tego stosunku, a na płaszczyźnie dyskursywnej - na niebezpieczeństwo utraty niezbędnej kompatybilności. W przedłożonym przyczynku 
identyfikacja stosunku między kulturą i techniką jako mediami zrównoważonego rozwoju została rozwinięta w trzyetapowym postępowaniu. Po pierwsze, rekonstruuje się strategię tematyzacyjną i refleksyjne oramowanie posługujące się środkami pojęciowej eksplikacji „kultury” i „techniki” i identyfikujące na jej podstawie wzajemne odniesienia momentów semantycznych i sensów zawartych w tych pojęciach. Po drugie, chodzi o inną strategię i inne oramowanie, które pyta o to, jak kultura i technika powstały i jakie funkcje spełniają, aby na tym tle rozszyfrować wzajemny między nimi stosunek. Po trzecie, kwestionuje się kulturę i technikę w aspekcie przypisywanych im własności bycia celem lub środkiem, a zamiast tego charakteryzuje się je jako „media” ludzkiej samorealizacji.

Słowa kluczowe: człowiek, życie, świat, przyroda, społeczeństwo, interakcja, komunikacja, sens, metabolizm.

\section{TECHNOLOGY AND CULTURE AS MEDIA OF SUSTAINABILITY - IS THERE A NEXUS BETWEEN HUMAN ECOLOGY, PHILOSOPHICAL ANTHROPOLOGY AND SOCIAL THEORY?}

The abundance of theming strategies and reflexions aimed at the identifikation of the relationship between culture and technology, reflects the complexity of the mutual conditionality and influences, interact with both phenomena. So far like the field of discussions of culture and art thus also extend, it is assumed that the relationship between theory and practice, they are based on a meaningful discourse between all approaches allows, regardless of from where how and by what means they develop this relationship mentally. It is certain, therefore, that it is both necessary and possible to focus attention on specific sections or characteristic compounds include, but - on the substantive level - to run the risk of not finding a significant access to this money, and without - on the discursive level - losing the necessary connectivity. In this paper, the discussion of the relationship between culture and technology is deployed as a media sustainability in a three-stage approach applied.Our discussion aims to unite considerations of philosophical anthropology and social theory to human ecology. It will be developed in three stages. The first section attempts to understand a strategy of thematization and a framework of reflection that are working with the instruments of conceptual explanations of culture and technology. The second will explore an other strategy of thematization together with an other framework of reflection which are asking how culture and technology have emerged and what functions they do fulfill, in order to decipher their relationship on this background. The third section will discuss culture and technology with regard to some properties attributed to them that are trying to prove their nature as ends and as resources, in order to prepare in this way the central thesis that is to characterize them as means of the self-realization of humankind in its ecological environment.

Keywords: Man, life, world, nature, society, interaction, communication, sense, metabolism.

DOI:10.7862/rz.2014.hss.41

Przesłano do redakcji: marzec 2014

Przyjęto do druku: październik 2014 